Supplementary Table 2 Oligonucleotides used in this study

\title{
Construction of strains
}

$\begin{array}{ll}\text { del-LMRG_02270-1A } & \text { AAAATCTAGAAGGGACGAGCTACCCATATA } \\ \text { del-LMRG_02270-1 B } & \text { GTCATCTTGCTTTTCTGTTTATAACTTCATATTGTGTCTCCCTCTAT } \\ \text { del-LMRG_02270-1 C } & \text { ATAGAGGGAGGACACAATATGAAGTTATAAACGAAAAGCAAGATGAC } \\ \text { del-LMRG_02270-1 D } & \text { AAAACCCGGGCCATCTGGTTCAAGTTCTGG } \\ \text { gInPQ complementation F } & \text { TTTTGGTACCGCTTATATTGCATACGCAAAAATG } \\ \text { gInPQ_complementation R } & \text { TTTTGAGCTCTGCTTTTGGAAATTCCAAAGTCATC } \\ \text { LMRG_02271E164AA } & \text { AAAATACGTAGTACTTGGACATGGCTAATCATC } \\ \text { LMRG_02271E164AB } & \text { GATCGAGGGCTGATGTAGGTGCATCGAACAACATAATGTCAGGATC } \\ \text { LMRG_02271E164AC } & \text { GATCCTGACATTATGTTGTTCGATGCACCTACATCAGCCCTCGATC } \\ \text { LMRG_02271E164AD } & \text { TTTTTACGTACTGGATGCTGTCGTAACAATTG } \\ \text { pPL2_yfp A } & \text { ATATGGGCCCGGGTTTCACTCTCCTTCTACATT } \\ \text { pPL2_yfp B } & \text { ACCTCCTTAGCTAGCAATGGCCCCCTCCTT } \\ \text { pPL2_yfp C } & \text { AAGGAGGGGGCCATTGCTAGCTAAGGAGGT } \\ \text { pPL2_yfp D } & \text { AATTCGGCCGTTAAAGCTTTTTATACAGTTCGT }\end{array}$


Supplementary Table 2 continued- Oligonucleotides used in this study

\section{SBP point mutation}

SBP-R105A

TGGCATGTCTATTACCGACGAAGCTAAACAAAAATTTGATTTCAGCG

SBP-R105A - RC CGCTGAAATCAAATTTTTGTTTAGCTTCGTCGGTAATAGACATGCCA

\section{Macrophage gene expression RT-qPCR}

IFN-B F

CCA AGA AAG GAC GAA CAT TCG

IFN-B R

CCG CCC TGT AGG TGA GGT T

GAPDH F

TTGTGGAAGGGCTCATGACC

GAPDH R

TCTTCTGGGTGGCAGTGATG

IL-6 F

TTCCATCCAGTTGCCTTCTTG

IL-6 R

GAAGGCCGTGGTTGTCACC

Bacterial gene expression RT-qPCR

rpoD F

GTGATCGGACGGTGAAGTTG

$r p o D R$

ATCGCACAAGAGCCAGTTTC

hly $F$

AAACACGCGGATGAAATCGA

hly $R$

TAACCTTTTCTTGGCGGCAC

plcA $F$

ATGTCCACGTCATGTCTCCG

plcA R

GGATGTCCGCTCTACCTGAC

$\operatorname{act} A F$

CGTCGTCGGATAGTGAGCTT

$\operatorname{act} A R$

CCACAACTGACTCTTTCGCC

plcB F

GCAACGGAAGACATGGTAGC

plcB R

TAGTCCGCTTTCGCCCTTTT 